\title{
LXXIII. The production of radium from uranium
}

\section{Frederick Soddy M.A.}

To cite this article: Frederick Soddy M.A. (1905) LXXIII. The production of radium from uranium , Philosophical Magazine Series 6, 9:54, 768-779, DOI: 10.1080/14786440509463329

To link to this article: http://dx.doi.org/10.1080/14786440509463329

$$
\text { 册Published online: } 15 \text { Apr } 2009 .
$$

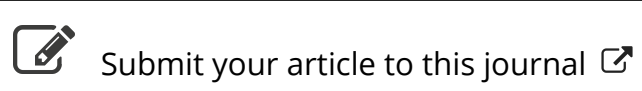

\footnotetext{
ЦIll Article views: 9
}

Q View related articles $₫$

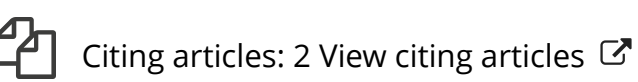




\section{$\left[\begin{array}{ll}768 & ]\end{array}\right.$}

\section{The Production of Radium from Uranium. \\ By Frederick Soddy, M.A. *}

SINCE the theory was brought forward that radioactivity $S$ is accompanied by the change of the atom of the radioelement (Rutherford \& Soddy, Phil. Mag. [6] v. 1903, p. 576), it has been recognized that the rate of change of radium is sufficiently rapid to cause the practically complete disappearance of any initial quantity of radium during the course of at most a few tens of thousands of years, so that the theory, if correct, must be able to account for the continued existence of this element. The rate of change of an element, like its atomic weight, seems to be a completely invariable and unvarying constant of nature, and there is no evidence to justify the view that the processes which accompany radioactivity have commenced at a late and recent period of the carth's history. On the other hand, the case of radium is essentially similar to that of all the other types of radioactive matter. It is changing much more rapidly than the elements uranium and thorium, but far slower than polonium. Any initial quantity of polonium would, according to the recent accurate determinations of the rate of change of this element (Ber. d. D. Chem. Gesell. 1905, p. 592) be reduced to less than one-tenth per cent. after the lapse of only five years, while the times for the same to occur in the case of the emanations of radium, thorium, and actinium respectively are 40 days, 10 minutes, and 37 seconds. To these cases, the idea of the comparatively recent origin of radioactive processes is of course inapplicable. From the first it was obvious that the conception of the continuous reproduction of the more rapidly changing types from the slower-changing socalled "parent-elements," which was arrived at by the study of the radioactive emanations and other similar types, embraced also the cases of radium and polonium. If, for example, radium and polonium are themselves intermediate or transition forms of elementary matter, which are being reproduced as fast as they themselves change, by the excessively slow disintegration of some parent element of higher atomic weight, not only the continued existence, but also the extremely minute quantity in which radium and polonium occur in nature is at once explained. As only two elements, thorium and uranium, are known with atomic weight greater than that of radium, the choice of a possible parent was

* Communicated by the Author. 
practically restricted to these two elements. Considerations analogous to those which indicated helium as one of the ultimate products of radioactive change, pointed strongly to uranium being the parent element of radium and polonium. The latter elements are only, to be found in the uranium minerals, and only those richest in the latter contain sufficient of the new elements to make the extraction profitable. Since radium and polonium are transition forms, they do not, like helium, accumulate to an indefinite extent with the lapse of time. In any disintegration series equilibrium is attained, and the relative quantities of the various members of the series do not further alter when certain definite proportions of the transition forms have accumulated. These proportions expressed in terms of the quantity of the parent element are the inverse ratios of the rates of change of the transition forms and parent elements respectively. From the relative radioactivity of uranium and radium, it followed that the rate of change of the former is about one-millionth of that of the latter, so that the quantity of radium accumulating in a mineral should be about one-millionth of the quantity of uranium present, and this ratio should be independent of the age of the mineral. This is approximately the value found experimentally by M. and Mme. Curie, and by Giesel, so that from the first there was a strong presumption in favour of the view that uranium in its own disintegration is continually producing radium. Rutherford has recently (Phil. Mag. [6] viii. 1904, p. 636) brought forward direct experimental evidence that polonium is one of the disintegration products of radium ; and in this paper similar direct experimental evidence is given to show that the radium itself results from the disintegration of uranium. The continuous disintegration series thus revealed, starting with the heaviest element known, embraces the majority of the known types of radioactive matter, and although direct experimental evidence is still lacking, probably ends, so far as the manifestation of radioactive phenomena is concerned, in the ultimate production of one of the heaviest non-radioactive elements, bismuth or lead. The time occupied in the passage, after the initial change of the uranium until the final change of polonium, during which the changing matter is present as intermediate forms, is a very considerable one, the average time being probably of the order of ten thousand years.

On this account experiments by a direct method, in which a reproduction of radium is looked for in uranium originally freed from that element, must be continued over a long 
period before a decisive result is attained. The experiments here described were started in May 1903. At first only negative results were obtained, but later, after a kilogram of uranium nitrate bad been kept under observation for a period of eighteen months, undeniable evidence of a positive character was obtained, although a much slower rate of production of radium from uranium was indicated than was to be expected if a direct change of uranium or uranium $\mathrm{X}$ into radium occurred. These two results were briefly announced in letters to 'Nature' (May 12th, 1904, and January 26th, 1905). Whetham ('Nature,' May 5th, 1904, and Feb. 2nd, 1905) has given the result of some experiments undertaken on the same problem, and was able to confirm the positive result announced in the second letter.

During the progress of these investigations, the problem has been attacked by a different method by other investigators, to whose work some reference will be made later in the paper. The method is based upon the accurate determination of the ratio of relative quantities of uranium and radium in a large number of minerals, to see whether the requirement that the two elements always exist in a fixed relative proportion is fulfilled.

Radium is distinguished from uranium in giving a relatively short-lived gaseous disintegration product or emanation, which is characterized by certain well-marked peculiarities. 'The emanation may therefore be used as a qualitative test for the presence of radium. Since the laws regulating the rate of production and accumulation of this emanation are well known (Rutherford \& Soddy, Phil. Mag. [6] v. 1903, p. 450), the amount of emanation accumulating in a known time can be used as a quantitative measure of the amount of radium present. The research divides itself into four sections. In the first place it will be shown that a quantity of uranium originally practically free from radium, and possessing therefore no power of producing an emanation, after being kept a certain time in a closed bottle develops an unmistakeable though still small emanating power. Secondly, the characteristics of the emanation produced will be shown to conform to those of the radium emanation. Thirdly, experiments will be given in which the quantity of emanation produced from the uranium was compared with the quantity produced from a known weight of radium, and the amount of radium finally present in the uranium deduced. Lastly, the probable cause of the slow rate of production will be considered. 
I.-Preliminary experiments with a hundred grams of commercial uranium nitrate showed evidence of the presence of radium in noticeable amount. It was found that the radium could be removed practically completely by repeated precipitation in its aqueous solution of small quantities of barium in the form of sulphate. The general method of testing for the presence of radium may be briefly described. The solution of uranium nitrate in water was kept for a period of at least some days in a closed bottle, into the mouth of which inlet and outlet tubes provided with taps had been ground. At the end of the period, which was accurately noted, a current of air was blown through the uranium solution, through a drying-tube containing calcium chloride between plugs of cotton-wool, into the electroscope. A volume of air sufficient to displace the whole of the air in the bottle, but smaller than the volume of the electroscope, was used. The electroscope was of the ordinary single-leaf type insulated by a sulphur bead, first described by C. T. R. Wilson, and the rate of discharge of the leaf was read by a microscope provided with a scale in the eyepiece. The natural leak of the instrument was always determined at the beginning of each experiment. The somewhat large natural rates of leak in the final series of measurements are due to the gradual accumulation of the later products of slow change left behind by the emanation. Rutherford has shown that this effect increases rather than decays with lapse of time. As, however, in every case the natural leak was small compared with the rate of leak measured, and as it remained constant throughout the day's experiments, it did not introduce any error into the measurements.

A kilogram of commercial uranium nitrate was dissolved in water, sulphuric acid was added and then successive small quantities of barium-nitrate solution, the liquid being left to stand, and filtered from the precipitated barium sulphate after each addition. The most effective conditions for the removal of the radium appeared to be to carry out the addition of barium drop by drop to the cold dilute solution of uranium nitrate, with constant stirring. Preliminary experiments indicated that the radium had been practically all removed, and on June 13th, after a final precipitation, the uranium solution was closed up in the bottle, and except for occasional tests, in which air was blown through, the solution remained undisturbed throughout the whole series of experiments. The initial observation was taken on June 20 th, 1903 , seven days after the closing of the bottle. The reading-microscope 
employed was one of home manufacture, and was only oneeighth of the magnifying power of that used in the final series of observations. These rates of leak have therefore been multiplied by eight to make them comparable with the later measurements. After blowing the air from the uranium solution into the electroscope, the rate of leak was increased from 0.048 division per minute (natural leak) to 0.136 division per minute. This is so small compared with the rates of leak ultimately obtained that it may be neglected. It represents less than $10^{-11}$ gram of radium.

Observations were taken occasionally over a period of eighteen months, and indicated a gradual growth of emanating power of the uranium solution. But, owing to the disturbing effects of the radium employed in other researches in the laboratory, the intermediate series of observations only possess a qualitative value. The final series was carried out in December 1904 and January 1905, in the newly erected chemical laboratory at the Glasgow University, before any radium had been introduced into the building. The first test was made on December 17th, 1904, the air not having been blown out of the bottle for nine months. An increase of the rate of leak of the instrument from 1.56 divisions per minute (natural leak) to 16.7 divisions per minute was obtained. Thus, after a period of about 500 days, the rate of leak due to accumulation of the emanation in the uranium solution was over a hundred times greater than that initially observed.

II.-The emanation from the uranium solution comported itself in all respects like the radium emanation, and there can be no doubt that the emanation resulted from radium produced from the uranium. The radium emanation when newly admitted into an electroscope, owing to the production of the excited activity, gives a rate of leak which steadily rises over the first five minutes and then remains comparatively steady during the next five minutes. If the emanation is then blown or sucked out and the rate of leak immediately tested, it will be found to be roughly equal to the amount by which the initial rate of leak increased. In the course of a few minutes after the removal of the emanation the rate of leak of the electroscope becomes very small, if the emanation has remained in the instrument only a few minutes. The actual measurements recorded in the test of the air from the uranium-nitrate solution on Dec. 17th 1904, are here given as a fairly typical example of what was always observed. 
Natural leak of electroscope 1.56 divisions per minute. Leak after the introduction of the air from the uranium solution:-

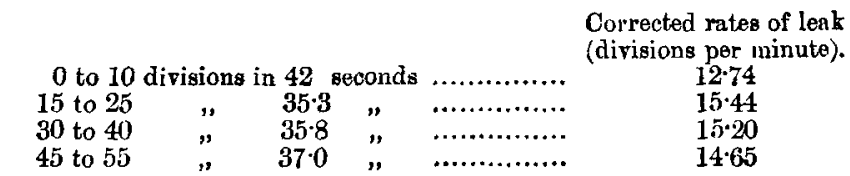

Electroscope recharged.

0 to 10 divisions in 38.4 seconds ..............

15 to $25 \quad " \quad 360 \quad, \quad \ldots \ldots \ldots \ldots \ldots . .15 .1$

30 to $40 \quad " \quad 370, " \quad \ldots \ldots \ldots \ldots \ldots . . . .14 \%$

Microscope reset.

0 to 20 divisions in $63 \cdot 2$ seconds ..............

25 to $35 \quad " \quad 28.4 \quad, \quad \ldots . . \ldots \ldots \ldots . . .19 .5$

Microscope reset.

0 to 10 divisions in 32 seconds $\ldots . . . \ldots . . . . \quad 17 \cdot 2$

The air in the electroscope was then sucked out:

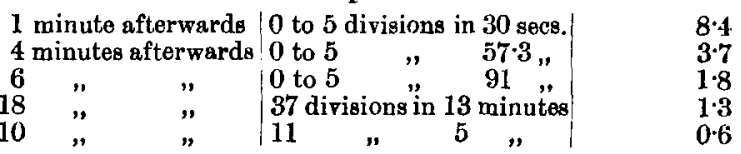

Similar results were always obtained when the emanation from the uranium was tested, and also when minute quantities of the radium emanation itself were introduced into the electroscope for the purpose of calibration.

A further test of the identity of the emanation from the uranium with that from radium was obtained by taking four series of observations in which the period of accumulation of the emanation from the uranium was varied. For the case of radium the quantity of emanation accumulating attains a practical maximum after three or four weeks, and generally if $I_{\infty}$ represents the rate of leak due to this maximum, and $\mathrm{I}_{t}$ that due to the quantity of emanation accumulating in $t$ seconds

$$
\frac{\mathrm{I}_{t}}{\mathrm{I}_{\infty}}=1-\epsilon^{-\lambda t}
$$

where $\lambda=2.10^{-6}$. The following results were obtained :-

\begin{tabular}{|c|c|c|c|c|}
\hline Date. & $\begin{array}{c}\text { Time of } \\
\text { accumulation. }\end{array}$ & Rate of Leak. & Natural Leak. & $\begin{array}{c}\text { Corrected } \\
\text { Rate of Leak. }\end{array}$ \\
\hline $\begin{array}{l}\text { Dec. } 17,1904, \ldots . . \\
\text { Dec. } 21,1904 \ldots \ldots \\
\text { Jan. } 7,1905, \ldots \ldots \\
\text { Jan. } 9,1905\end{array}$ & $\begin{array}{l}9 \text { months } \\
4 \text { days } \\
17 \text { days } \\
53.5 \text { hours }\end{array}$ & $\begin{array}{r}16 \cdot 7 \\
12 \cdot 0 \\
18 \cdot 0 \\
9 \cdot 5\end{array}$ & $\begin{array}{l}1 \cdot 56 \\
1 \cdot 8 \\
2 \cdot 35 \\
2 \cdot 8\end{array}$ & $\begin{array}{c}15 \cdot 1 \\
10 \cdot 2 \\
15 \cdot 65 \\
6 \cdot 7\end{array}$ \\
\hline
\end{tabular}


The rates of leak are given in divisions of the scale in seconds, and were those obtained after the emanation had been left in the electroscope about 5 minutes, that is, at the end of the initial period of rapid increase. It may be pointed out that it is impossible to obtain strictly comparable measurements of the quantity of radium emanation with the electroscope. No attempt was made to arrive at more than the order of magnitudes measured. If the observation of January 7 is selected as the most accurate of the series, and the rates of leak theoretically to be expected for the other periods of accumulation calculated from it by means of the equation given, the observed results are found to be in fair agreement with the theoretical.

\begin{tabular}{|r|c|c|c|}
\hline & Time of accumulation. & Observed. & Calculated. \\
\cline { 1 - 3 } I. ............... & 9 months & $15 \cdot 1$ & $16 \cdot 5$ \\
II. ............... & 4 days & $10 \cdot 2$ & $8 \cdot 2$ \\
III. .............. & 17 days & $15 \cdot 65$ & 15.65 \\
IV. ............... & 52.5 hours & 6.7 & $5 \cdot 3$ \\
\hline
\end{tabular}

The agreement is as close as can be expected and, taken in conjunction with the whole behaviour of the emanation, leaves no doubt as to its identity with that of radium.

III.-The quantity of emanation observed in the experiments recorded after a period of eighteen months' production of radium is far below that which is to be expected on the assumption that the radium atom results directly by the change of the uranium $X$ atom. The diagram (fig. 1) represents the course of change in a quantity of uranium represented by $10^{11}$ atoms for 150 days. Time in days is ploted on the horizontal axis, while the figures on the vertical axis denote numbers of atoms. The straight line $A B$ represents the number of atoms of uranium undergoing change, primarily into uranium $X$ atoms. The curve ACD represents the number of atoms of radium that would be formed if the uranium $\mathrm{X}$ atom changed directly into the radium atom. The area $A B D C$ gives the number of uranium $X$ atoms present at any instant. AE is the average life of the uranium $\mathrm{X}$ atom. The rate of change or radioactive constant of uranium is taken to be $6 \times 10^{-17}$ (secs. ${ }^{-1}$ ), and is derived from that of radium by comparing the relative 
$\alpha$ radioactivity of the two elements and the number of changes in which $\alpha$ rays are expelled in the two cases. The quantity of radium actually produced even after 500 days is far too

Fig. 1.

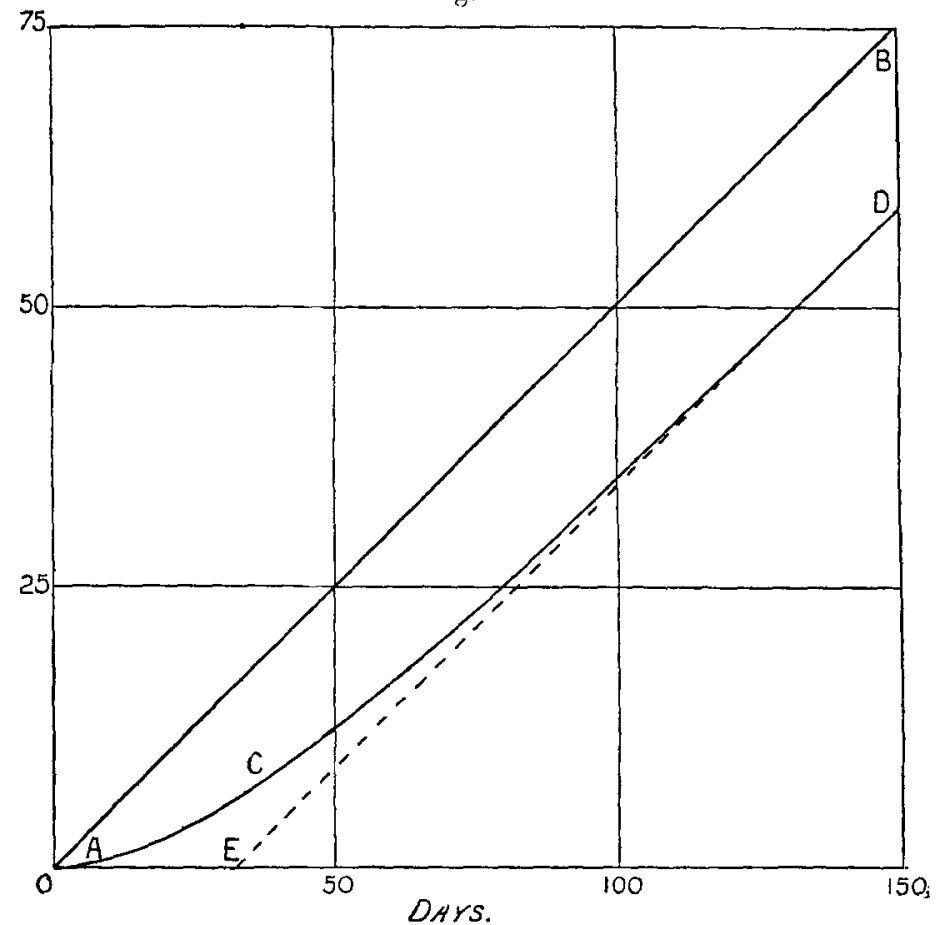

small to be represented on the diagram, and as a matter of fact amounts to about one atom of radium for every $3 \times 10^{11}$ atoms of uranium.

This result was arrived at in the following manner:-A quantity of Giesel's purest radium bromide weighing $1 \cdot 1$ milligrams was dissolved in water in a small closed test-tube, into which inlet and outlet tubes provided with taps were sealed. The emanation accumulating for a known time was swept out by a current of air into a gas-burette, and the volume measured. After thorough mixing of the contents of the gas-burette a small known volume was delivered into a second gas-burette, again diluted to a known volume, mixed. and a small known fraction delivered into a third burette, The dilution was performed so that about one-millionth of the original emanation was finally obtained and delivered 
into the electroscope. Care was taken to use fresh burettes, rubber tubing, mercury, \&c. for each dilution, as it was found that otherwise the results were quite untrustworthy. In one experiment, the emanation had been allowed to accumulate for 16 days in the radium solution. In this time about 94 per cent. of the maximum or equilibrium quantity accumulates. It was dilnted with air to 73 c.c. and 0.71 c.c. taken. This was diluted to 92 c.c. and 0.042 taken. The last quantity was made up to 50 c.c. and 20 c.c. introduced into the electroscope. After 5 minutes the corrected rate of leak was 24 divisions per minute.

Several determinations gave results of the same order of magnitude, althqugh the individual determinations varied over an extreme range of 1 to 4 . From the mean of the results it was deduced that the equilibrium quantity of emanation from about $10^{-10}$ gram of radium (element) caused a leak of 1 division of the scale per minute. 16.5 divisions per minute may be taken as the measure of the equilibrium amount of emanation accumulating in the uranium solution after 567 days. This corresponds to the presence of $1.6 \times 10^{-9} \mathrm{gram}$ radium. If the uranium nitrate is assumed to contain 50 per cent. of uranium, and the radioactive constant of the latter is taken as $6 \times 10^{-17}\left(\mathrm{sec}^{-1}\right)$, then, on the assumption that there is a direct change of uranium into radium, the quantity of the latter that would be produced from a kilogram of uranium nitrate in 567 days is $1.5 \times 10^{-6} \mathrm{gram}$. The effect of the uranium $\mathrm{X}$ stage would be practically inappreciable after a period of one and a half years. So that the result shows that the production of radium from uranium during the first one and a half years is only about one thousandth of the quantity calculated on the assumption of a direct change.

IV.-From the figure already given it will readily be seen that the effect of the uranium $X$ stage is to reduce the initial rate of production of radium below the final rate during an initial period corresponding in order of magnitude to the period of average life of the uranium $X$ atom. The same consideration would hold true for any other unknown intermediate products between uranium $\vec{X}$ and radium. So that the low result obtained would be explained if one or more intermediate forms existed between uranium $\mathrm{X}$ and radium, for which the period of average life was large compared with the time of experiment. Before considering some independent evidence in favour of this view, another possibility has first to be considered. It seemed not unlikely that the radium, under the conditions in which it was produced, 
was in the non-emanating form, so that the emanation was retained and did not escape freely from the solution. In the purification process an excess of sulphuric acid had been added, and some still remained in the solution after the final precipitations with barium. The radium therefore would be present as sulphate and it is well known that this compound obstinately rotains the emanation. Mme. Curie has stated in her Thesis that the activity of radium sulphate is unchanged by shaking with water for a whole day.

To test this possibility the uranium solution was removed from the bottle, a small quantity of barium nitrate solution was added drop by drop, and the precipitated sulphate filtered off. This precipitate should have contained the greater part of the produced radium. It was ignited, and any emanation evolved sent into the electroscope. No great quantity of emanation was, however, given off. The barium was then got into solution by first boiling with sodium carbonate solution, washing the barium carbonate produced, and dissolving it in hydrochloric acid. The process was repeated until the whole of the barium (and radium) was obtained in solution. The uranium solution was treated with excess of barium to free it completely from sulphuric acid, concentrated and returned to the bottle. Tests were then carried out on the amount of emanation accumulating both in the uranium and in the barium solutions. That accumulating in the former amounted to about one-third, and that accumulating in the latter to about two-thirds of the amount obtained from the uranium before treatment, and no rapid increase of the emanating power of the uranium solution was observed for the first few days. There are therefore no grounds for believing that the low rate of production was due to the radium not giving up its emanation, or that more radium was present than the original experiments indicated.

With regard to the hypothesis of intermediate forms of relatively slow rates of change, it is possible, owing to some recent results, to approach the question on more general grounds than formerly. It is clear that as polonium and radium are to be regarded as products of uranium, the other radio-elements, and especially actinium, present in pitchblende probably have a similar origin, and may in fact be the missing intermediate forms. From Rutherford's recent investigations (Phil. Mag. [6] viii. 1904, p. 636 and 'Nature,' Feb. 9th 1905) it appears probable that polonium is the product of the seventh change of the radium atom, and that four out of the seven changes are accompanied by the expulsion of the heavy a particle. In a recent paper by Marckwald on radio- 
tellurium (Ber. d. D. Chem. Gesell. 1905, p. 591) fairly conclusive evidence of a chemical nature is brought forward that polonium (radio-tellurium) stands to tellurium in the periodic table in a similar relation to that in which radium stands to barium. It therefore immediately follows bismuth, the atomic weight of which is $208.5(0=16)$, and the atomic weight of polonium may be estimated with fair probability as being in the neighbourhood of 212. Thus in the change of the radium atom (225) into polonium, four $\alpha$ particles are expelled and a reduction of atomic weight amounting to about 13 units occurs, so that the view that the $\alpha$ particle is or becomes an atom of helium seems to be fairly well borne out. In the change of the atom of uranium (238) into radium a similar reduction of atomic weight of 13 units takes place, and it may therefore be expected that about four a particles are expelled in this case also. By analogy with other disintegration series, it is to be expected that in addition to the $\alpha$-ray changes several rayless and $\beta$-ray changes occur also, and of the latter one, the change of uranium into uranium $\mathbf{X}$, is in fact known. The hypothesis that there are several slow-period intermediate changes between uranium and radium gains in definiteness by these considerations.

The results obtained in the investigations of the ratio in which radium and uranium are contained in the natural minerals, which have been recently published, bear out this hypothesis in the strongest possible manner. Since the drafting of the present paper Boltwood (Phil. Mag. [6] ix. 1905, p. 599) has published analyses of 22 minerals containing very varying proportions of uranium, and finds in each case the expected proportionality to exist between the quantities of the two elements. McCoy (Ber. d. D. Chem. Gesell. 1904, p. 2641) has examined the radioactivity of several minerals containing uranium, and has shown that in those in which thorium is not present the activity is proportional to the quantity of uranium present. This supports the view that radium, actinium, and polonium are all intermediate products in the disintegration of uranium. An interesting speculation arises as to the nature of the final product in the disintegration series. After the change of polonium, in which $\alpha$ rays only are expelled, no further changes accompanied by the expulsion of radiant particles occur. If the atomic weight of polonium is in fact 212, the expulsion of one a particle from the atom would lower the atomic weight further to about 208. If this change is in finct the last of the series, the ultimate product must accumulate 
indefinitely in the minerals, and therefore be a known element. The choice it would seem must rest between bismuth (208.5) and lead (206.9). Boltwood calls attention to the persistent appearance of lead as a constituent of the uranium-radium minerals, and cites certain evidence in favour of the view that it is one of the final products. Owing to the rapid rate of change of polonium, and to the refined methods of separating it from the mineral on a large scale introduced by Marckwald, the experimental identification of the ultimate product would seem to be now mainly a matter of cost.

\section{An Optical Paradox.}

By Lord RAYLEIGH, O.M., F.R.S.*

CONSIDER the following combination:-A point source A of approximately homogeneous light $(\lambda)$ is focused by the lens LL upon the object-glass of a telescope T. In its

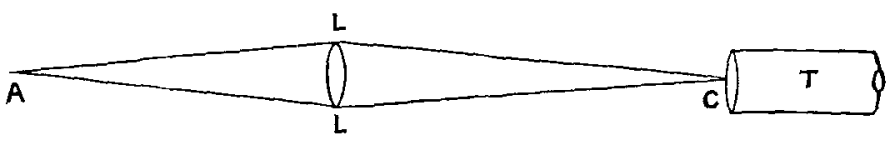

turn the telescope is focused upon L. According to geometrical optics the margin of the lens L should be seen sharp by an eye applied to the telescope; but when we consider the limitation of aperture at the object-glass of the telescope, we come to the conclusion that the definition must be very bad. The image of $\mathrm{A}$ at $\mathrm{C}$ constitutes the usual diffraction pattern of which most of the light is concentrated in the central disk. The diameter of this disk is of the order $\lambda$. LC/LL. If this be regarded as the effective aperture of $T$, the angular resolving power will be found by dividing $\lambda$ by the above quantity, giving $\mathrm{LL} / \mathrm{LC}$; so that the entire angular magnitude of the lens LL is on the limits of resolving power.

If this be admitted, we may consider next the effect of enlarging the source A, hitherto supposed to be infinitely small. If the process be carried far enongh, the objectglass of $\mathrm{T}$ will become filled with light, and we may expect the natural resolving power to be recovered. But here we must distinguish. If the enlarged source at $\mathrm{A}$ be a selfluminous body, such as a piece of white-hot metal or the carbon of an electric arc, no such conclusion will follow. There is no phase-relation between the lights which act at

* Communicated by the Author. 